\title{
Reliable dynamic monitoring of bridges with integrated GPS and BeiDou
}

\author{
Ruijie Xi ${ }^{\mathrm{a}, \mathrm{b}}$, Hua Chen ${ }^{\mathrm{a}, \mathrm{c}^{*}}$, Xiaolin Meng ${ }^{\mathrm{b}}$, Weiping Jiang ${ }^{\mathrm{a}, \mathrm{d}}$, Qusen Chen ${ }^{\mathrm{a}, \mathrm{b}}$ \\ a School of Geodesy and Geomatics, Wuhan University, 129 Luoyu Road, Wuhan 430079, Hubei, China; \\ ${ }^{\mathrm{b}}$ Nottingham Geospatial Institute, The University of Nottingham, Nottingham NG7 2TU, United Kingdom; \\ ${ }^{\mathrm{c}}$ Key laboratory of Geospace Environment and Geodesy, Ministry of Education, Wuhan University, 129 Luoyu \\ Road, Wuhan 430079, Hubei, China. \\ ${ }^{\mathrm{d}}$ GNSS Research Center, Wuhan University, 129 Luoyu Road, Wuhan 430079, Hubei, China.
}

\begin{abstract}
GPS (Global Positioning System) in recent years has been widely used for the measurement of deflections of bridges. However, due to multipath and satellite signal obstructions, caused by towers, cables and passing vehicles, the reliability of deformation monitoring with GPS is still a problem. Recent research with respect to multi-GNSS (Global Navigation Satellite System) technology, though, have proved to enhance satellite visibility and availability for positioning, navigation and timing (PNT) for users. Its benefits involving application in bridge monitoring are still rarely studied. In this paper, we propose a composite strategy where integrated GPS and BDS (BeiDou Navigation Satellite System) dual-frequency carrier phase data processing is carried out to improve the reliability of bridge monitoring with GNSS measurements. In addition, SNR (signalto-noise ratio) based stochastic model and post-fit residual editing strategies are utilized to enhance the reliability further. In a group of fixed point experiments, improvements of $20 \%$ to $30 \%$ in precision were achieved with the integrated GPS and BDS compared to GPS-only results. Based on the real GPS and BDS measurements collected on the Baishazhou Yangtze River Bridge in China, we assessed the performance of the proposed method. In the vibration experiment, no apparent effects on natural frequencies identification were found by introducing BDS into the solution at ideal observation environment. However, the combined GPS and BDS results seem to be much more promising, with lower background noise. Meanwhile, the integrated GPS and BDS data processing with post-fit residual editing and SNR-based stochastic model strategies can effectively deal with satellite signal obstruction and the influence of multipath effect to attain reliable dynamic deformation monitoring information for bridges.
\end{abstract}

Keywords: Dynamic bridge deformation monitoring; Integrated GPS and BDS; Multipath effect, Reliability 


\section{Introduction}

Global Positioning System (GPS) as a technique that was developed from 1970s, has gradually become a major means used in structural bridge deformation monitoring in recent years, due to its continuous, all-weather, automated and highly accurate measurement services. With the continuous development of hardware and software, especially the increasing sampling rate, GPS begins to show its unique advantages in the aspect of the bridge dynamic monitoring (Meng et al. 2007). In the last years, a large number of achieves on deformation monitoring of structural bridges using GPS has been published (Meng et al. 2007; Yu et al. 2014; Moschas and Stiros 2011; Han et al.2016; Chan et al.2006; Meng et al.2003; Meng et al.2004; Psimoulis et al.2008; Wang et al. 2016; Yi et al.2013). For instance, Meng et al. 2007 based on the field tests of the response of the Wilford Bridge in the UK, has confirmed the GPS is a viable tool for both structural deflection monitoring and natural frequency detection. Yu et al. 2014 carried out a series of monitoring experiments on the Wilford suspension bridge and confirmed the modal frequencies of the bridge can be accurately identified from GPS measurements, and successfully validated by those from the accelerometer data. Moschas and Stiros (2011) reveal the potential of GPS to measure the displacement history and the modal frequencies of a short-span pedestrian bridge, i.e. modal frequencies up to $1 \mathrm{~Hz}$ and displacements of around 10-20 mm. Han et al. (2016) reported the deformation accuracy with the amplitude of several millimeters can be successfully detected, and the spectrum of the pylon response under typhoon loading obtained from GPS data. Peppa et al. (2017) used the spectral content of the signalto-noise ratio (SNR) of GPS signals to detect vibration frequencies of bridges.

However, to date, there are still many problems to solve with GPS to provide reliable monitoring information for the structural bridge. Due to the obstructions, such as cables and passing vehicles, the observation environment on the bridge is not always good enough for GPS observation, especially for the GPS antenna on the bridge deck (Wieser and Brunner 2002; Moschas and Stiros 2014; Moschas et al. 2013). The obstructions may dramatically reduce the number of observed GPS satellites and weaken the satellite geometry. In case of disturbed tracking condition, the system will not be available or make a high position dilution of precision (PDOP) value in positioning. Multipath effect and signal diffraction results from obstructions can also degrade the precision and accuracy of GPS or produce unreliable and even failure of monitoring results, and can adversely 
affect the ambiguities resolution in data processing (Wang et al. 2017). Some literatures reported using fusion of GPS and GLObal NAvigation Satellite System (GLONASS) data could enhance the number of tracking satellites and geometry, and using sidereal filter or analogous methods could deal with multipath. Nevertheless, due to the frequency division multiple access (FDMA) signal mode of GLONASS system, the ambiguity of GLONASS is difficult to be fixed (Li and Zhang 2014). The dynamic multipath caused by passing vehicles cannot be eliminated by a sidereal filter or other types of digital filters (Moschas and Stiros 2014). In case of severe multipath, the ambiguity parameters tend to be fixed to wrong integer values, which will probably induce biases in the monitoring results. The large noise, at the same time, can over flood the true movement and limit the GPS vibration monitoring in model frequencies identification. Hence, these issues are still critical in dynamic deformation monitoring of the bridge with GPS.

Fortunately, except for the GPS and GLONASS system, BeiDou Navigation Satellite System (BDS) and Galileo (satellite navigation) are in the process of providing global coverage services. Better signal quality, similar Code division multiple access (CDMA) mode and different constellations provided by BDS and Galileo can be a way to enhance satellite visibility and availability for positioning, navigation and timing (PNT) for users (Yang et al. 2011).

BDS is a global satellite navigation system, which is independently developed, deployed, and operated by China and still in progress until to 2020 (Yang et al. 2011; Shi et al. 2012). Up to now, there are 23 satellites in BDS constellation and it is capable of providing PNT services in AsiaPacific Region. Three constellation types of BDS satellites are considered, including Geostationary Orbit (GEO), Inclined Geosynchronous Orbit (IGSO), and Medium Earth Orbit (MEO). The geodetic reference system used by BDS is the China Geodetic Coordinate System 2000 (CGCS2000), and its definition is: the origin is located at the mass center of the earth, the Z-axis is in the direction of the IERS Reference Pole (IRP), the X-axis is directed to the intersection of IERS reference meridian (IRM) and the plane passing through the origin and normal to the Z-axis. The Yaxis, together with Z-axis and X-axis, constitutes a right-handed orthogonal coordinate system. It is an earth-centered earth-fixed (ECEF) system. The origin of the CGCS2000 system is also the geometric center of the CGCS2000 ellipsoid, and the Z-axis coincides with the semi-minor axis of the CGCS2000 ellipsoid. The parameters of the CGCS2000 ellipsoid and WGS-84 (World Geodetic 
System 1984) are as Table 1. As can be seen, only $1 / f$ is slight different between the two ellipsoids. According to Gao et al. (2012), the differences of $1 / f$ between the two ellipsoids can be ignored for short baseline RTK positioning, and the coordinate difference between BDS and GPS systems can be neglected.

Table 1 The ellipsoid parameters of CGCS 2000 and WGS84.

\begin{tabular}{cll}
\hline Ellipsoid Parameters & CGCS2000 & WGS84 (1150) \\
\hline$G M\left(10^{14} \mathrm{~m}^{3} \mathrm{~s}^{-2}\right)$ & 3.986004418 & 3.986004418 \\
$\left(10^{-5} \mathrm{rad} \mathrm{s}^{-1}\right)$ & 7.292115 & 7.292115 \\
$a\left(10^{6} \mathrm{~m}\right)$ & 6.378137 & 6.378137 \\
$1 / f\left(10^{2}\right)$ & 2.98257222101 & 2.98257223563 \\
\hline
\end{tabular}

As an important member of Global Navigation Satellite System (GNSS) community, BDS has all the advantages that GNSS have, and is capable of providing similar precision and stability (Jiang et al. 2017; Xi et al. 2017; Xi et al. 2015; Xiao et al. 2016). It is shown that the precision of BDS carrier phase and code measurements are approximately $2 \mathrm{~mm}$ and $33 \mathrm{~cm}$, respectively, which are comparable to those of GPS. The radial precision of the BDS satellite orbit determination is better than $10 \mathrm{~cm}$, which confirms that BDS positioning precision and applications are comparable to that of GPS (Shi et al., 2013; Yang et al., 2014). Jiang et al. (2017) show that with the current in-orbit BDS satellites, in the mid-latitude of China, accuracy of $1 \mathrm{~mm}$ in horizontal component and 2-3 $\mathrm{mm}$ in vertical component can be achieved respectively. In bridge monitoring area, the performance of BDS in terms of precision and vibration monitoring has also been confirmed to be comparable with GPS (Xi et al. 2017; Roberts and Tang 2016). Recent studies state that the combined solution of GPS and BDS can improve the availability of positioning and enhance the positional precision with clean data (Msaewe et al. 2017; Deng et al. 2014). However, structural health monitoring (SHM) of bridges with integrated GPS and BDS are still rarely studied.

This paper aims at developing a new integrated GPS and BDS data processing method to improve the precision and reliability of dynamic bridge monitoring. The methods used to decrease the impact of multipath to displacements and the ambiguity resolution are developed and introduced in this paper. The paper is divided into four sections: Section 1 is introduction. Section 2 formulates the data processing method; Section 3 includes the experiments and results analysis; Section 4 draws 
the conclusions.

\section{Methodology}

\subsection{Observation model}

In the most general terms of using GPS or BDS for SHM of bridges, monitoring stations located at the feature points of bridges, and one or more reference stations are set at stable points, not far from the bridge. In operation, these stations receive the satellite signal simultaneously and the data processing software will process the data in real-time. In GPS or BDS observations, as pseudorange is easily contaminated by multipath and hardware delays, biases are significant. Therefore, in our data processing module, we only use the dual-frequency carrier phase observations. The pseudorange observations are used to calculate the receiver clock error in the stage of getting satellite positions and cycle slip detection (Liu 2013). For a satellite $i$ observed by receiver $p$, the dual-frequency GPS or BDS phase observation model can be presented as follows:

$$
\left\{\begin{array}{l}
L_{1, p}^{i}=\rho_{p}^{i}+c\left(d t_{p}-d t^{i}\right)+T_{1, p}^{i}-I_{1, p}^{i}+\lambda_{1}\left(N_{1, p}^{i}+B_{1, p}-B_{1}^{i}\right)+\varepsilon_{1, p}^{i} \\
L_{2, p}^{i}=\rho_{p}^{i}+c\left(d t_{p}-d t^{i}\right)+T_{2, p}^{i}-I_{2, p}^{i}+\lambda_{2}\left(N_{2, p}^{i}+B_{2, p}-B_{2}^{i}\right)+\varepsilon_{2, p}^{i}
\end{array}\right.
$$

where $L$ is the carrier phase observation; $\rho$ is the geometric distance between satellite $i$ and receiver $p ; c$ is the speed of light; $d t_{p}$ and $d t^{i}$ are the clock errors of receiver and satellite, respectively; $T_{p}^{i}$ and $I_{p}^{i}$ denotes the slant tropospheric and ionospheric delays; $\lambda_{1}$ and $\lambda_{2}$ are the dual-frequency wavelength of carriers phase; $N$ is the carrier phase ambiguity; $B_{p}$ and $B^{i}$ are receiver-dependent and satellite dependent carrier phase hardware delay, respectively; and $\varepsilon$ is the measurement noise of the carrier phase. It should be noted that the equations here are only appropriate for CDMA systems.

Since the baselines are usually short (usually $<5 \mathrm{~km}$ ), double difference (DD) method can eliminate the satellite-dependent terms (such as satellite clock offsets, receiver clock errors and carrier phase hardware delay) and the distance-dependent terms (such as tropospheric and ionospheric delay). Then, with reference station $q$ and pivot satellite $j$, the DD models can be read: 


$$
\left\{\begin{array}{l}
\delta L_{1, p q}^{i j}=\delta \rho_{p q}^{i j}+\lambda_{1} \delta N_{1, p q}^{i j}+\delta \varepsilon_{1, p q}^{i j} \\
\delta L_{2, p q}^{i j}=\delta \rho_{p q}^{i j}+\lambda_{2} \delta N_{2, p q}^{i j}+\delta \varepsilon_{2, p q}^{i j}
\end{array}\right.
$$

where $\delta$ operator represents the two-station DD between two observed satellites. For the purpose of fast ambiguity fixing, the ambiguity parameters of second frequency (N2) can be represented as the combination of first frequency (N1) ambiguity and wide-lane (WL) ambiguity

$$
\delta N_{2}=\delta N_{1}-\delta N_{w l}
$$

After the linearization and considering Eq. (3), Eq. (2) is reorganized in matrix form as

$$
\delta l=\left[\begin{array}{c}
\delta L_{1, p q}^{i j}-\delta \rho_{p q, 0}^{i j} \\
\delta L_{2, p q}^{i j}-\delta \rho_{p q, 0}^{i j}
\end{array}\right]=\left[\begin{array}{ccc}
G & \lambda_{1} & 0 \\
G & \lambda_{2} & -\lambda_{2}
\end{array}\right]\left[\begin{array}{c}
X \\
\delta N_{1, p q}^{i j} \\
\delta N_{w l, p q}^{i j}
\end{array}\right]+\left[\begin{array}{c}
\delta \varepsilon_{1, p q}^{i j} \\
\delta \varepsilon_{2, p q}^{i j}
\end{array}\right]
$$

where $\delta \rho_{p q, 0}^{i j}$ is the geometric distance calculated by provided stations' initial coordinates and $\delta l$ is the Observations minus Computations (OMC) terms of dual-frequencies, $X$ denotes the baseline component parameters. $G$ is the corresponding geometry matrices containing the satellite-to-receiver unit vector.

As for GPS and BDS system, they both apply CDMA technology. The model described above is available for the two GNSS systems. Let $S_{G}$ and $S_{C}$ denote the number of GPS and BDS satellites that are tracked by the two receivers. Since GPS and BDS do not share common frequencies, the double differences for two systems are calculated using a separate pivot satellite in the integrated model. The system of linearized double-difference GNSS observation equations in Eq. (4) can be written in the form

$$
y=B b+A a+\varepsilon
$$

where $y=\left[\delta l_{G}^{T}, \delta l_{C}^{T}\right]^{T}$ contains the double differenced OMC, $\delta l_{*} \in \square^{\left(S_{*}-1\right) \times 2} ; b$ represents the three baseline components; and $a=\left[a_{G}^{T}, a_{C}^{T}\right]^{T}$ contains the carrier phase integer ambiguities $a_{*} \in \square^{\left(S_{*}-1\right) \times 2}$. The ambiguity design matrix is given by

$$
A=\operatorname{blkdiag}\left(\Lambda_{G}, \Lambda_{C}\right)
$$

with $\Lambda_{*}=\left[\begin{array}{cc}\lambda_{*, 1} & 0 \\ \lambda_{*, 2} & -\lambda_{*, 2}\end{array}\right] \otimes I_{S_{*}-1}$, where $\lambda_{*}$ denotes the wavelength of dual-frequency for GPS 
and BDS, $I_{S_{*}-1}$ a unit matrix of size $S_{*}-1$, and $\otimes$ is the Kronecker product. With the double differenced geometry matrices $G_{*} \in \square^{\left(S_{*}-1\right) \times 3}$ containing the satellite-to-receiver unit vector, matrix $B$ follows as

$$
B=\left[\begin{array}{c}
e_{2} \otimes G_{G} \\
e_{2} \otimes G_{C}
\end{array}\right]
$$

where $e_{2}$ denotes the two vectors of ones.

\subsection{Stochastic model}

$\varepsilon$ in Eq. (5) is assumed as a zero-mean Gaussian noise with the covariance matrix

$$
Q=Q_{l}+Q_{b}
$$

where $Q_{l}$ denotes the single differenced measurement noise of un-pivot satellite $i$ between reference $(p)$ and monitoring station $(q)$, and $Q_{b}$ is the single differenced measurement noise of pivot satellite $j$ between the two stations. Assuming that all measurements of the two receivers are uncorrelated, $Q_{l}$ and $Q_{b}$ are given

$$
\left\{\begin{array}{l}
Q_{l}=I_{2} \otimes I_{S_{*}-1} \otimes\left[\left(\sigma_{p}^{i, *}\right)^{2}+\left(\sigma_{q}^{i, *}\right)^{2}\right] \\
Q_{b}=I_{2} \otimes O_{S_{*}-1} \otimes\left[\left(\sigma_{p}^{j, *}\right)^{2}+\left(\sigma_{q}^{j, *}\right)^{2}\right]
\end{array}\right.
$$

where $O_{S_{*}-1}$ denotes a matrix of size $\left(S_{*}-1\right) \times\left(S_{*}-1\right)$ with all values of $1 . \sigma^{2}$ is the oneway covariance for carrier phase observations.

In GNSS data processing, elevation-dependent stochastic models are often used to weight undifferenced phase noise. It assumes the effects of multipath as well as atmospheric errors are elevation-dependent. However, in bridge monitoring, the serious multipath and signal diffraction effect often occurred even in high elevation satellites, due to the obstructions, such as towers, cables and passing vehicles, around the antenna. Fortunately, the power of a GNSS signal is a measurement of its quality. One way of expressing the GNSS signal power, used by most receiver brands, is the carrier-to-noise power-density ratio (C/N0). It is the ratio of the signal carrier to noise power in a 1Hz bandwidth (Brunner et al. 1999; Hartinger and Brunner 1999). The SNR-based weight scheme (i.e. SIGMA- $\Delta$ model) is reasonable for the one-way carrier phase observations. Therefore, in order 
to reduce the influence of high multipath effect, the one-way covariance can be calculated as:

$$
\sigma^{2}=\sigma_{0}^{2}\left[1+C\left\lfloor 10^{-\left(C / N 0_{\text {measured }}\right) / 10}\right]\right.
$$

where the factor $C$ consists of the carrier loop noise bandwidth and a conversion term from cycle ${ }^{2}$ to $\mathrm{mm}^{2}$. In this paper, for first frequency $C=0.00224 \mathrm{~m}^{2}$ and second frequency $C=0.00077 \mathrm{~m}^{2}$, as mentioned in Dai (2008). Normally, the value of $\sigma_{0}$ is selected to $3 \mathrm{~mm}$ for GPS and BDS carrier phase observations (Amiri-Simkooei and Tiberius 2007).

\subsection{Parameter estimation}

A Kalman filter is applied for parameter estimation in this study, because it is efficient and suitable for real-time positioning with a large number of states and observations. The state vector $X_{k}$ contains coordinates of monitoring station and the ambiguity parameters at epoch $k$. Based on the cooperative dynamic model, the matrix form of the system state equation is given by

$$
X_{k+1}=\Phi_{k+1, k} X_{k}+F w_{k}
$$

where $\Phi_{k+1, k}$ is the state transition matrix. In this paper, it is an identity matrix. $F$ is the coefficient matrix of system noise, and $w_{k}$ is the system noise vector.

Because the pseudorange measurements are not used in this paper, the prior covariance matrix constraints for the coordinate and ambiguity parameters, must be given, that is $C_{k}$, as

$$
C_{k}=\operatorname{blkdiag}\left(I_{3} \otimes \sigma_{p}^{2}, I_{\left(S_{*}-1\right) \times 2} \otimes 10000.00\right)
$$

where $\sigma_{p}$ is the initial prior standard deviation for baseline component parameters. Normally, the initial position of monitoring station is set to its approximate coordinate. The initial prior standard deviation should be set according to the accuracy of the approximate coordinate. The initial values of ambiguity parameters were zero and the initial prior standard deviations were set to 100 cycles, assuming the large number part of ambiguities had been removed by Geometry-Free (GF) and Melbourne-Wübbena (MW) combinations (Chen 1998).

\subsection{Ambiguity fixing strategy and post-fit residual editing}

After parameter estimating with a Kalman filter, the coordinate parameters with float ambiguities can be obtained. Then the least-squares ambiguity decorrelation adjustment (LAMBDA) method will be applied to fix the ambiguities (Teunissen, 1995). Since the long wavelength for WL 
ambiguities, in this paper, the GPS and BDS WL ambiguities will be fixed together forward and then the N1 ambiguities. In order to ensure the ambiguity resolution (AR) reliability, the solved integer ambiguities will be validated with the well-known ratio test. The threshold is set to 2.0 as many scholars did.

However, during the data processing, the multipath effect resulting from passing vehicles and stay-cables may be serious in some cases. The ambiguities may pass the ratio test and be fixed to an incorrect integer value. In this case, we will get the wrong baseline estimation. In this paper, a post-fit residual editing strategy is employed to test the validity of the fixed ambiguities. If the postfit residuals after ambiguity resolution surpass 0.35 (empirical value), the corresponding ambiguities will be set to unfixed and reprocess the data at current epoch (Deng et al. 2014).

Beside the method mentioned previously, additional processing options should be taken care are listed in Table 2.

Table 2 Additional data processing models and strategies used in this paper.

\begin{tabular}{ll}
\hline Options & Processing Strategy \\
\hline Ephemeris & GPS and BDS broadcast ephemeris \\
Signals & GPS L1/L2, BDS B1/B2 \\
Elevation cutoff angle & $10^{\circ}$ \\
Troposphere modeling & Corrected with GPT2w model (Lagler et al. 2013) \\
Coordinate system difference between GPS and BDS & Neglected in short baselines (Gao et al. 2012) \\
Time system difference between GPS and BDS & BDST=GPST+1356weeks-14seconds (Gao et al. 2012) \\
\hline
\end{tabular}

\section{Preliminary analysis}

\subsection{Software development}

Based on the theory described above, a home-made GNSS data processing software specialized in bridge deformation monitoring was developed, called GNSSDEM (GNSS DEformation Monitoring). It permits the users to select to process the data with GPS-only, BDS-only or integrated GPS and BDS (GPS+BDS). Different data processing strategies toward stochastic model, threshold of ratio and post-fit residual editing after ambiguity fixing can also be set by users. The software supports Receiver INdependent EXchange format (RINEX) files and real-time binary data stream, 
consisting of the State Space Representation (SSR) messages in the RTCM-3 (Radio Technical Commission for Maritime Services) format accessed via NTRIP (Networked Transport of RTCM via Internet Protocol) streams (Weber and Mervart 2012; Martin et al. 2015). The work flow chart of the data processing is shown in Fig 1.

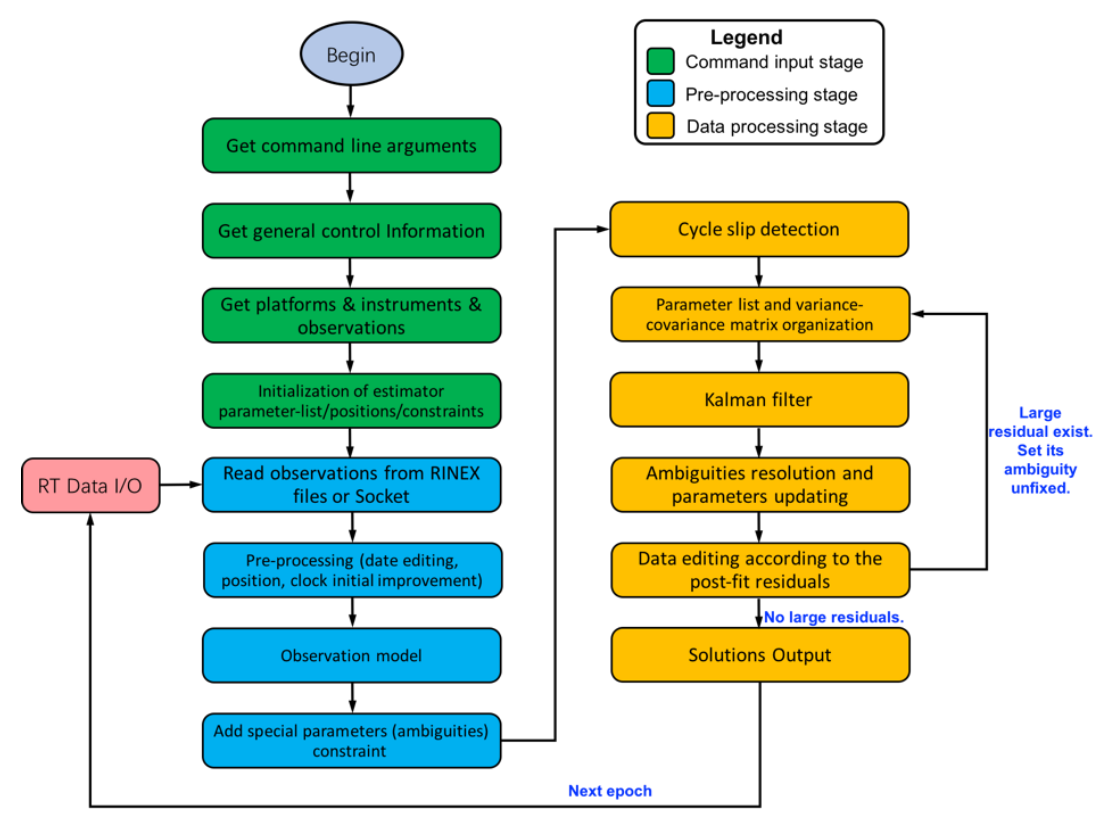

Fig 1 The work flow chart of GPS+BDS data processing.

\subsection{Ambiguity resolution performance analysis}

In this section, a group of GPS and BDS data collected from two short baselines formed by a reference station (called JZ01) and two monitoring stations (JC01 and JC02) were processed to validate the ambiguity resolution and precision performance of the proposed method. The stations were settled in Zhengzhou City, Henan Province, China, and the baseline lengths are $280.89 \mathrm{~m}$ and $162.96 \mathrm{~m}$ for JC01_JZ02 and JC02_JZ01 respectively. All stations were equipped with a TRIMBLE NETR9 receiver and a CHOKE RING antenna (TRM59900.00). The observation environment is quite well, no obvious shelters could be found around the stations. The sampling rate is $15 \mathrm{~s}$ and the data length is for one day (August $8^{\text {th }}$ 2014). Before the data processing, we used the GAMIT (GPS Analysis at MIT) software to obtain the coordinates of stations at centimeter level (Herring et al. 2015). Then, the data of GPS-only, BDS-only and GPS+BDS were processed separately with the proposed method to get the time series. The initial prior standard deviation for baseline component parameters were set to $3 \mathrm{~m}$. The ambiguity parameters including GPS ambiguities and BDS ambiguities for GEO, IGSO and MEO satellites were all estimated and searched by LAMBDA 
method.

Firstly, the BDS-only, GPS-only and GPS+BDS data processing were performed in the instantaneous mode to examine the single-epoch ambiguity resolution performance, where the ratio of epochs with correctly resolved ambiguities with respect to the number of all processed epochs is used as an index for validation. In the approach, the data of one epoch is resolved independently with no precious epochs' solutions accumulated. After data processing, we calculated the percentages of ambiguity resolution ratio whose values are greater than 2,5 , and 10 , which are shown in Fig 2.
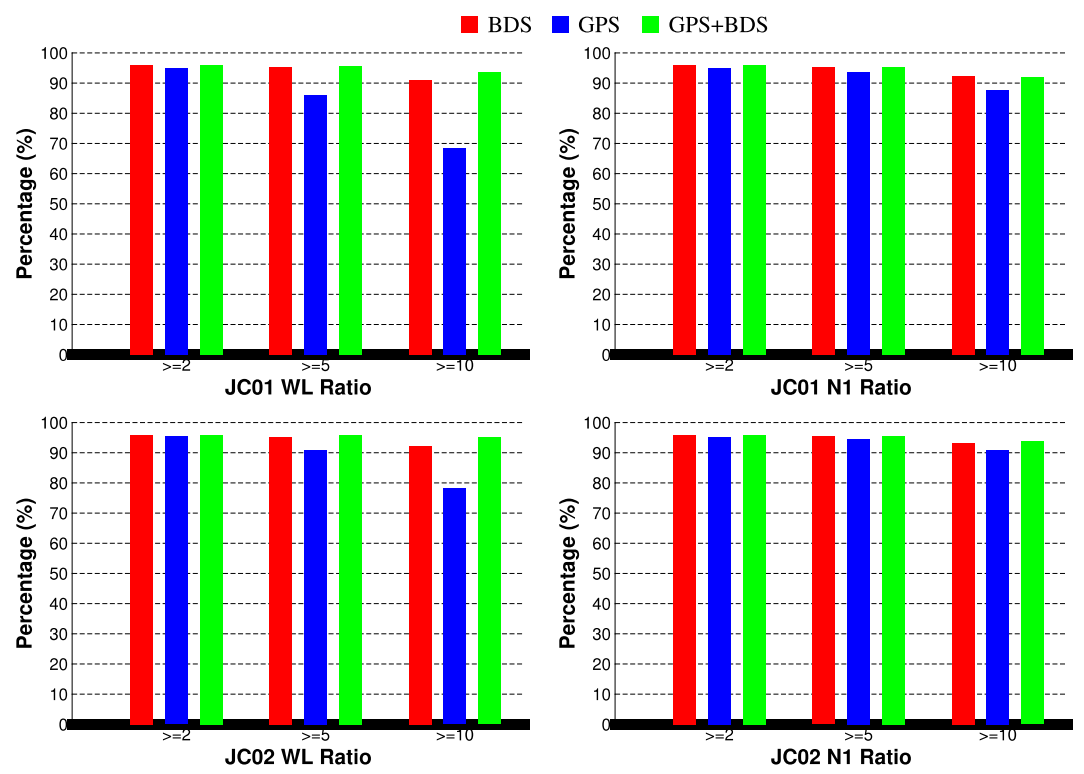

Fig 2 Percentage of single-epoch ambiguity resolution ratio values for BDS-only, GPS-only, and GPS+BDS.

It shows that the percentage of epochs with correctly resolved WL and NL ambiguities are all around $95 \%$ when the threshold is set to 2 . The GPS+BDS system is slightly higher than the BDSonly and GPS-only counterparts. Meanwhile, over $90 \%$ epochs whose WL ambiguity ratio is greater than 5 and 10 for BDS-only and GPS+BDS systems. However, the GPS-only system shows a slightly lower percentage. After WL ambiguities are correctly resolved, approximately $90 \%$ epochs can fix the NL ambiguities with a high ratio value. Therefore, for short baselines, if there are no obvious shelters around the station, GPS and BDS ambiguities can be easily fixed, with about 95\% single-epoch ambiguity success rate for ratio threshold of 2 .

\subsection{Precision analysis}

As a matter of fact, in GPS and BDS data processing, once the ambiguities are reliably fixed, 
the integer ambiguities will be passed to the following epochs until the cycle slip occurs. Then, the fixed solutions are achieved. Fig 3 presents the position errors for BDS-only, GPS-only and GPS+BDS fixed solutions at baselines JC01_JZ01 and JC02_JZ01.
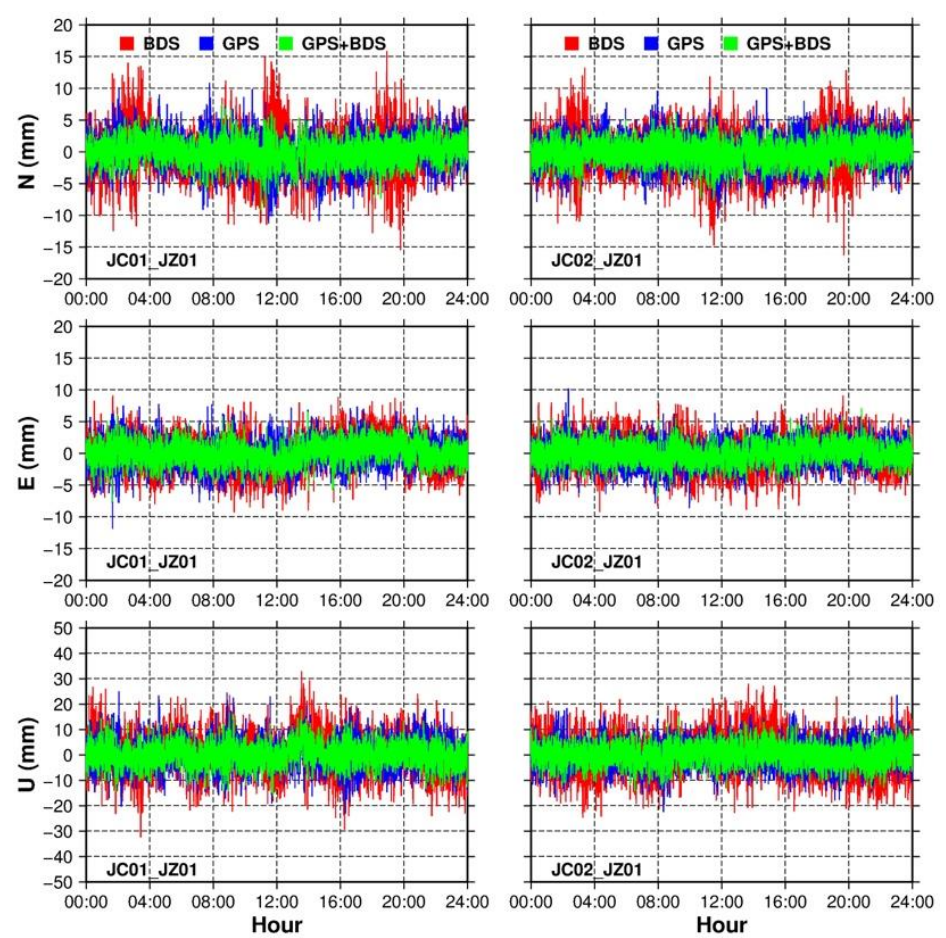

Fig 3 BDS-only, GPS-only and GPS+BDS data processing results of JC01_JZ01 and JC02_JZ01.

From Fig 3, we can see that, the variation of BDS-only is larger than GPS-only under most circumstances, and GPS+BDS results always show a best result, mostly within only $5 \mathrm{~mm}$ in North and East directions and $10 \mathrm{~mm}$ in Up direction. The standard deviation (STD) of BDS-only, GPS-only and GPS+BDS results are listed in

Table 3. From STD values, the precision of BDS-only results is worse than that of GPS-only, and the GPS+BDS results show the highest precision, with better than $2 \mathrm{~mm}$ in horizontal component and $5 \mathrm{~mm}$ in vertical component, and compared with GPS-only results, the precision is improved by about $20 \%-30 \%$ in all directions. As known, the precision improvement is profit from more observations and the enhancement of satellite geometry.

Table 3 STD values of BDS-only, GPS-only and GPS + BDS residuals.

Improvement

BDS (mm) GPS (mm) GPS+BDS (mm) compared with GPS

\begin{tabular}{lllllllllllllll}
$\mathrm{N}$ & $\mathrm{E}$ & $\mathrm{U}$ & $\mathrm{N}$ & $\mathrm{E}$ & $\mathrm{U}$ & $\mathrm{N}$ & $\mathrm{E}$ & $\mathrm{U}$ & $\mathrm{N}$ & $\mathrm{E}$ & $\mathrm{U}$ \\
\hline
\end{tabular}




\begin{tabular}{lllllllllllll}
\hline $\mathrm{JC} 01$ & 3.6 & 2.7 & 7.8 & 2.6 & 2.2 & 5.7 & 1.9 & 1.7 & 4.5 & 26.9 & 22.7 & 21.1 \\
$\mathrm{JC} 02$ & 3.3 & 2.6 & 7.6 & 2.4 & 2.0 & 5.1 & 1.7 & 1.6 & 4.1 & 29.2 & 33.3 & 19.6 \\
\hline
\end{tabular}

Fig 4 presents the numbers of BDS, GPS and GPS+BDS satellites and their corresponding PDOP values. We can see that, compared with the standalone system, the observations of GPS+BDS are doubled and the PDOP value is stable which is lower than 2.

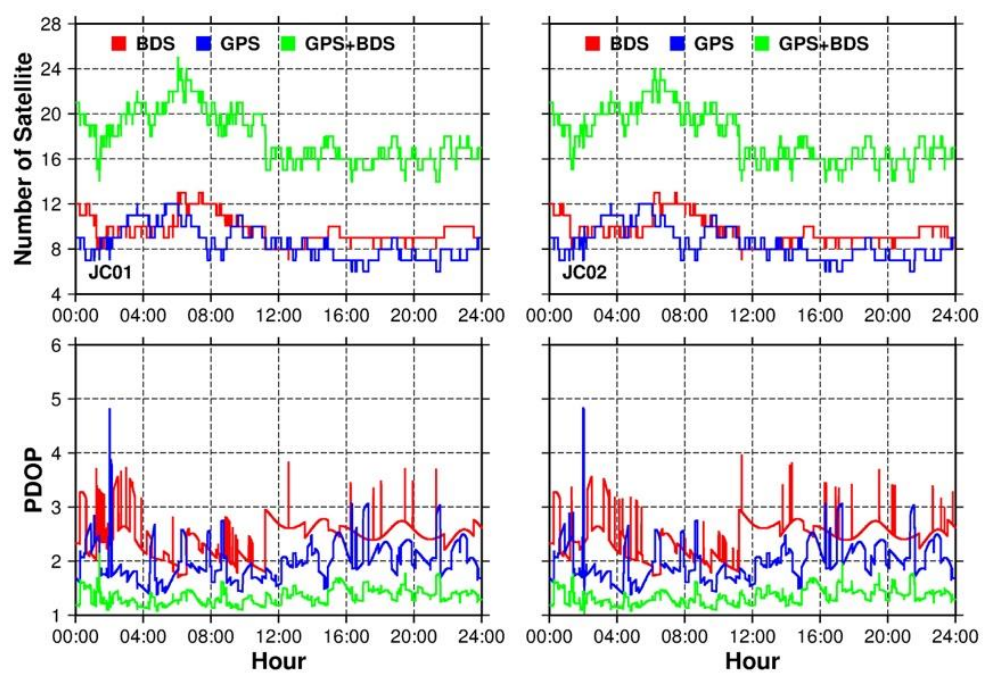

Fig 4 Number of BDS, GPS and GPS+BDS satellites that can be tracked in JC01 and JC02 (August $8^{\text {th }}$ 2014), and corresponding PDOP values.
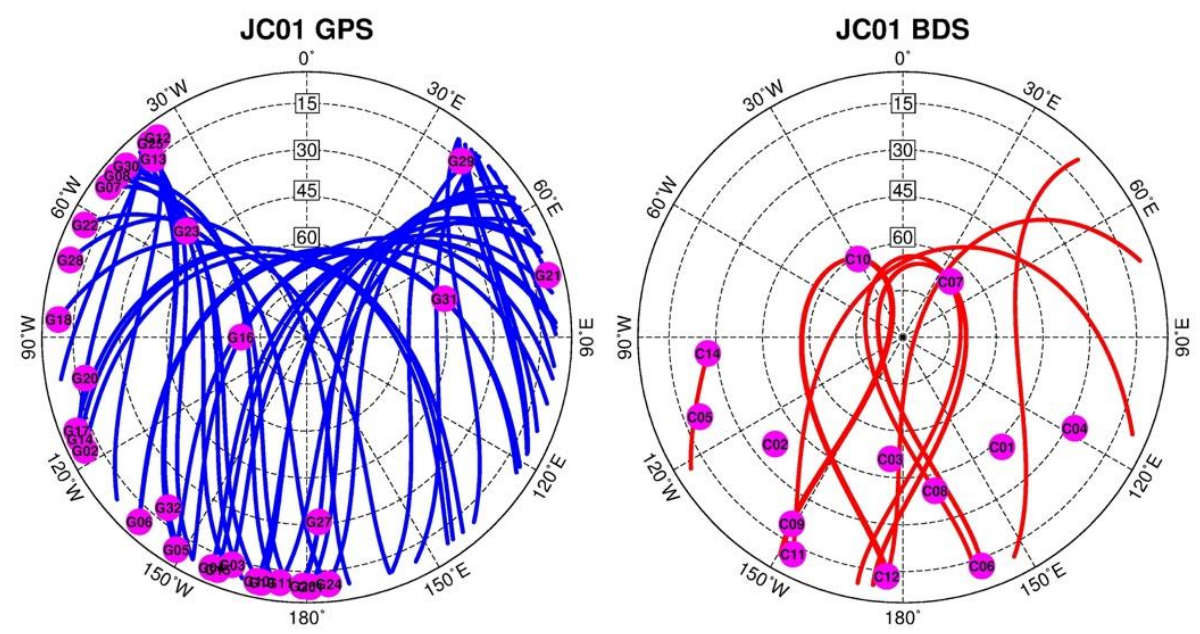

Fig 5 Sky plot of GPS and BDS satellites in JC01 with a $10^{\circ}$ elevation cutoff angle.

It should be noticed that, the observed satellite numbers for GPS and BDS are at the same level during the whole day. However, BDS shows a higher PDOP value than GPS. Fig 5 gives the sky plot of GPS and BDS satellites respectively. From Fig 5, we know that, it is because most satellites are in the southern side of station mostly. The satellite geometry is not good for BDS compared with GPS constellation. That is also the reason that the precision of BDS results are lower than GPS, especially in North and Up directions. Meanwhile, when there is a sudden growth of BDS PDOP 
value, a great residual error will be shown in Fig 3.

To better understand the spectral characteristics of the above results in the frequency domain, the BDS-only, GPS-only and GPS+BDS time series were analyzed using Fast Fourier Transform (FFT) spectral technique. Fig 6 is presented the spectra of the BDS-only, GPS-only and GPS+BDS time series. Since the sampling rate is only $15 \mathrm{~s}$, the highest frequency can only reach to $1 / 15 \mathrm{~Hz}$.

It is found that BDS-only time series appear having strong colored noise in very low frequencies $(<0.0005 \mathrm{~Hz})$. Then, GPS-only solutions show a high level of white noise in frequency band from $0.0005 \mathrm{~Hz}$ to $0.01 \mathrm{~Hz}$. In the high-frequency bands, BDS-only and GPS-only results both show slightly high white noise. For the GPS+BDS time series, it is apparent that the combined GPS and BDS observations can reduce the noise level in all frequency bands and the characteristic of the noise is closer to white noise.
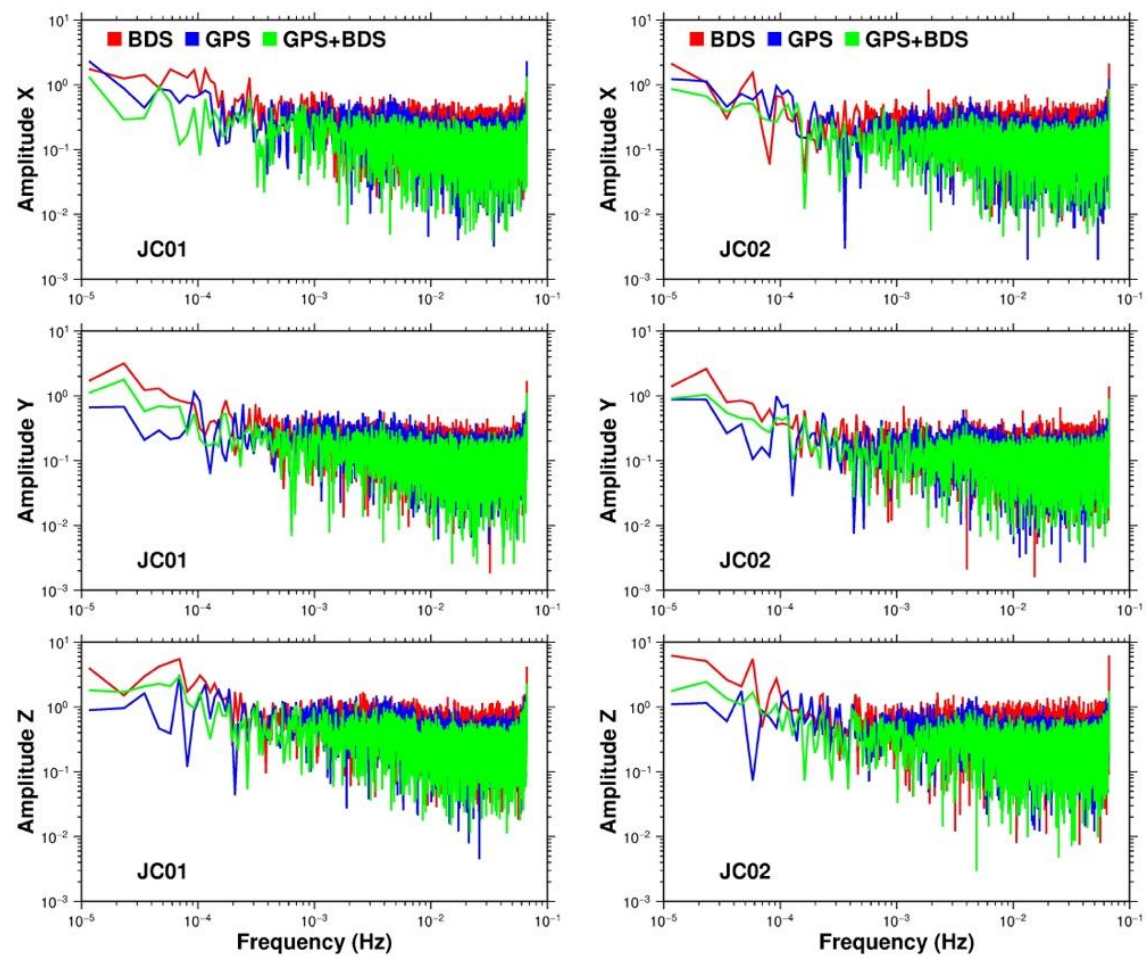

Fig 6 Spectral analysis for BDS-only, GPS-only and GPS+BDS solutions.

\section{Bridge experiments and results analysis}

The GNSS observations used in this paper were collected from the Baishazhou Yangtze River Bridge. The Baishazhou Yangtze River Bridge is a highway bridge over the Yangtze River in Wuhan, China. It was designed by China Railway Major Bridge Reconnaissance and Design Institute (BRDI) from 1997. It is 3,586 meters long and 28.5 meters wide with the main span of 618 meters. It has 
six lanes and a capacity of 50,000 vehicles a day.

In order to comprehensively monitor the state of the bridge, the monitoring sites located on the middle span, top of tower, girder boxes are selected. The reference station is located on the northeast of the bridge approximately $2 \mathrm{~km}$ away from the middle span of the bridge. The distribution of experimental monitoring stations on the bridge is shown in Fig 7.

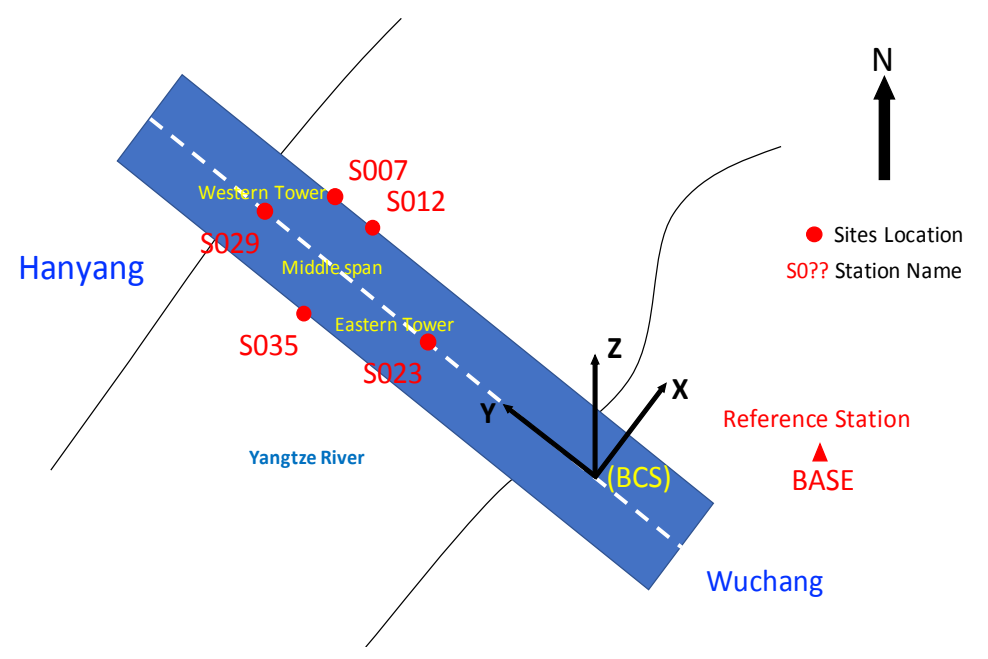

Fig 7 Distribution of experimental tracking stations on the bridge. Bridge Coordinate System (BCS) is also defined in the figure. (Red points are locations of antennas of monitoring stations. The four characters are the monitoring station names.)

At each station, ComNav GPS and BDS receiver board (ComNav-K508) and antenna are used in this field experiment. The experiment was carried out on September $27^{\text {th }} 2016$, and lasted 16 hours. The sampling rate for each site is set to $10 \mathrm{~Hz}$ and the elevation cutoff angle is $10^{\circ}$. The details of the monitoring stations are given in Table 4.

Table 4 The details of the monitoring stations.

\begin{tabular}{cccc}
\hline Station Name & Location & Baseline Length $(\mathrm{m})$ & Sampling Rate $(\mathrm{Hz})$ \\
\hline S012 & Middle Span & 2480.45 & 10 \\
S035 & Middle Span & 2476.09 & 10 \\
S023 & Eastern Tower & 2173.45 & 10 \\
S029 & Western Tower & 2789.58 & 10 \\
S007 & Box Girder & 2733.65 & 10 \\
\hline
\end{tabular}

\subsection{Vibration monitoring}

In dynamic monitoring of bridges, deflections and modal frequencies of the middle span and 
supporting towers are important parameters for cable-stayed or suspension bridges. In this paper, two stations were mounted at the both side of the middle span (S012 and S035) and another two located at top of the towers (S023 and S029). To validate the performance of the method proposed above in vibration monitoring, we used the GNSSDEM software to process the data of BDS-only, GPS-only, and GPS+BDS separately of the four stations for one hour (12:00-13:00, September $27^{\text {th }}$ 2016). Before the data processing, GAMIT software was applied to obtain the initial coordinate of the reference station. The initial coordinates of monitoring stations were then calculated by code differential positioning with the code measurements of the reference station and monitoring stations. Since the baseline is short, the accuracy of code differential positioning can achieve to decimeter level. However, the monitoring stations are always moving, and always within $5 \mathrm{~m}$. Then, we set the initial prior standard deviation of the coordinate of monitoring stations to $5 \mathrm{~m}$.
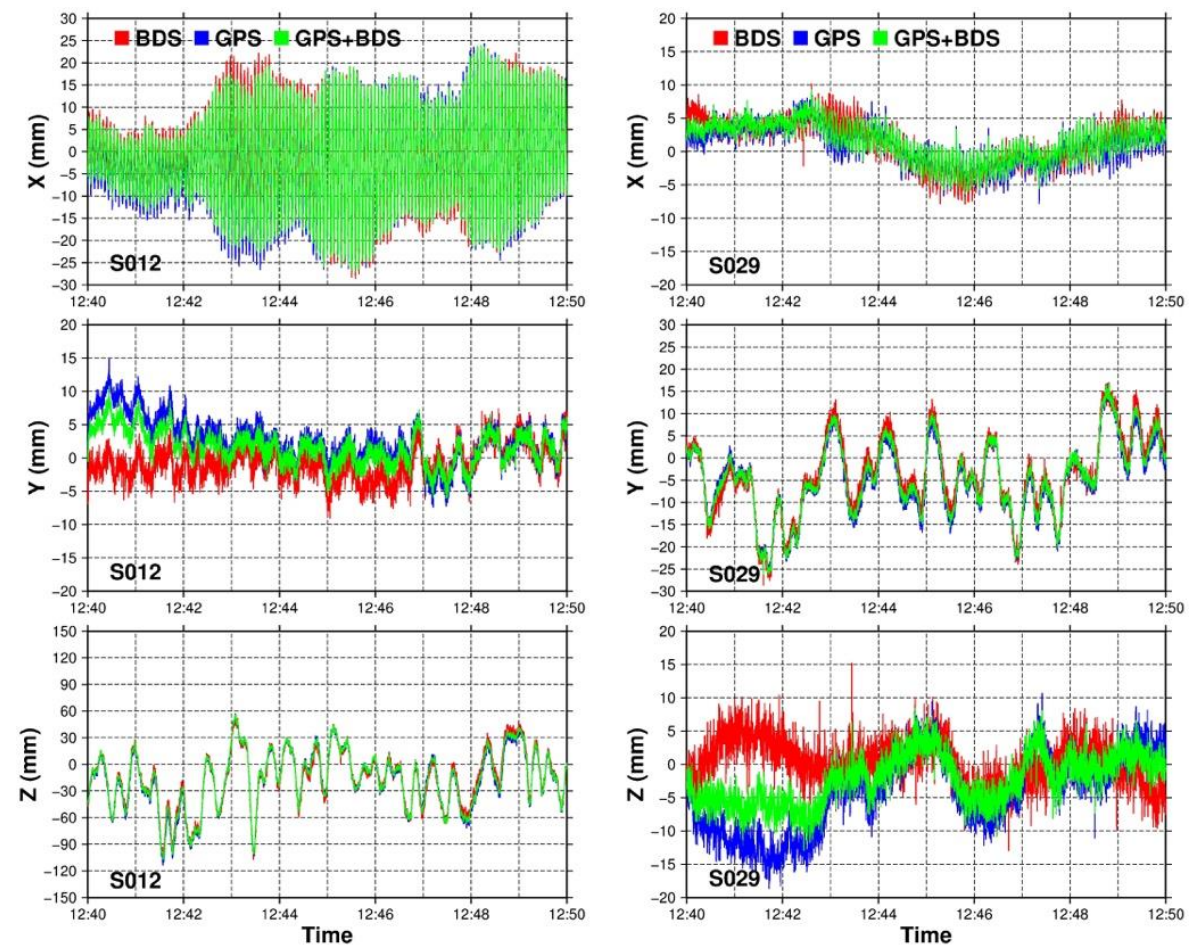

Fig 8 Vibration time series of S012 and S029 (12:40-12:50, September 27 2016$)$.

Fig 8 denotes the vibration time series of S012 at the middle span and of S029 at top of the western tower. Since S035 and S023 show the same results with S012 and S029 respectively, their results are not shown here. Due to the high sampling rate $(10 \mathrm{~Hz})$ and huge data quantity, results for only 10 minutes (12:40-12:50) were shown. In order to visualize the movement of the bridge, we have transformed the positioning solution in WGS84 system to BCS. For the vibration time series 
in Fig 8, the results from the three data sources are almost the same. Roughly speaking, the GPSonly and BDS-only are good enough to obtain the vibrations and displacements. That means, at the good observation environment, the combined GPS and BDS strategy cannot improve the performance at a large extent (Roberts and Tang 2016). However, the GPS+BDS results seem to be much more promising.

Fig 9 presents the spectral analysis of S012 and S029 for one hour. Apparently, the GPS+BDS time series show a lower noise in all frequency bands. GPS seems has a higher noise level in low frequencies $(<0.5 \mathrm{~Hz})$, and BDS has a higher noise in high frequency bands $(>0.5 \mathrm{~Hz})$. The reasons of this phenomenon should be investigated further. Meanwhile, from Fig 9, the mode frequencies can be found from the time series of the three systems, and the values are same for the different systems. Table 5 listed the designed Finite Element Model (FEM) parameters for the Baishazhou Yangtze River Bridge by Zhang et al. (1998), and the experimental value obtained with GPS measurements from Huang et al. (2005). Apparently, the natural frequency we got are comparable to the designed values and tested ones from other research.
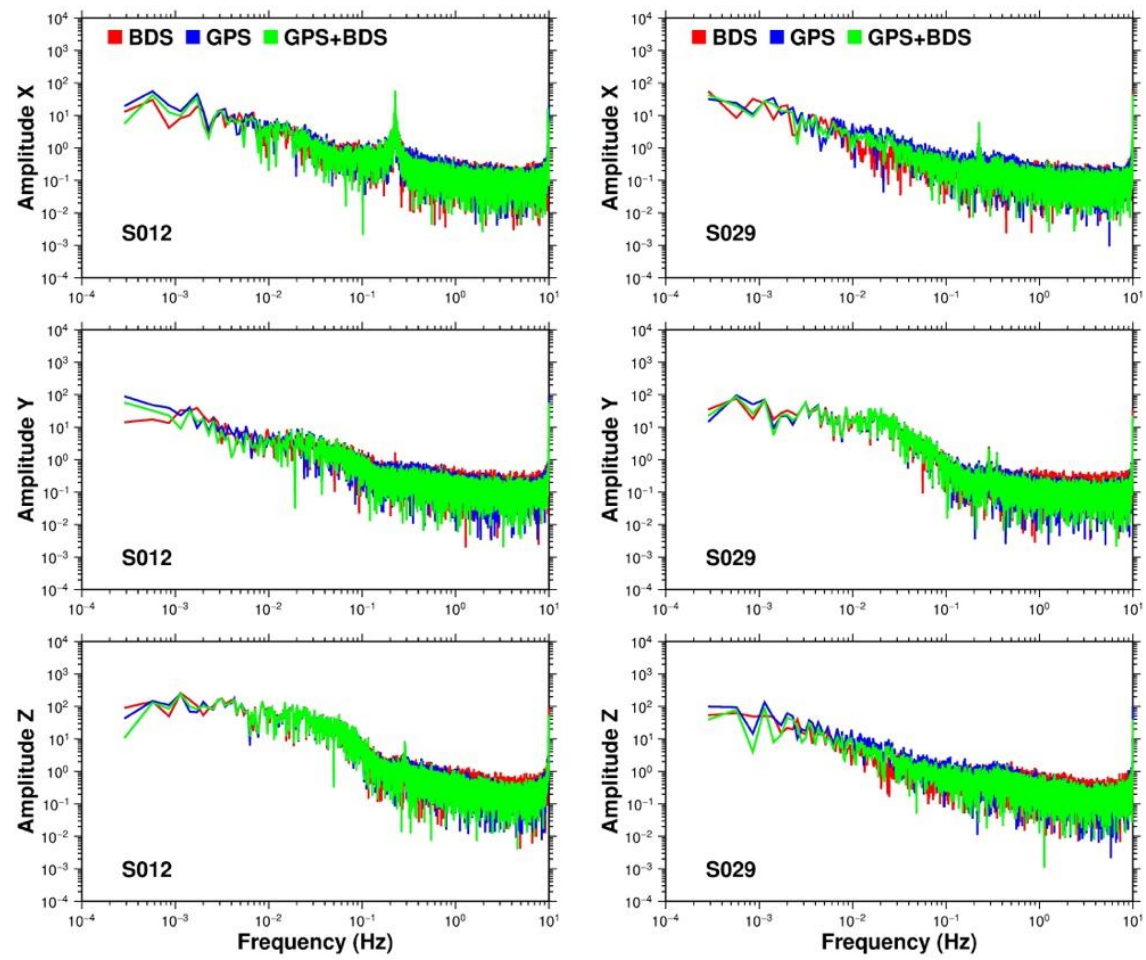

Fig 9 FFT spectra of S012 and S029 time series (12:00-13:00, September $\left.27^{\text {th }} 2016\right)$.

Table 5 Historical modal frequencies of Baishazhou Yangtze Bridge found in literature research. 


\begin{tabular}{cccc}
\hline Zhang et al. (1998) & $0.213 \mathrm{~Hz}$ & $0.283 \mathrm{~Hz}$ & $0.336 \mathrm{~Hz}$ \\
Huang et al. (2005) & $0.229 \mathrm{~Hz}$ & $0.293 \mathrm{~Hz}$ & - \\
This study & $0.226 \mathrm{~Hz}$ & $0.288 \mathrm{~Hz}$ & $0.351 \mathrm{~Hz}$ \\
\hline
\end{tabular}

\subsection{Availability and reliability}

As mentioned in Section 1, towers, intensive cables and passing vehicles beside the monitoring stations can obstruct GNSS signals. The obstructions mainly cause severe multipath effect, signal diffraction and satellite exclusion, which will adversely affect the performance of GNSS deformation monitoring in the aspect of availability and reliability. In this section, an example of obstruction test will be given to validate the performance of the proposed method.
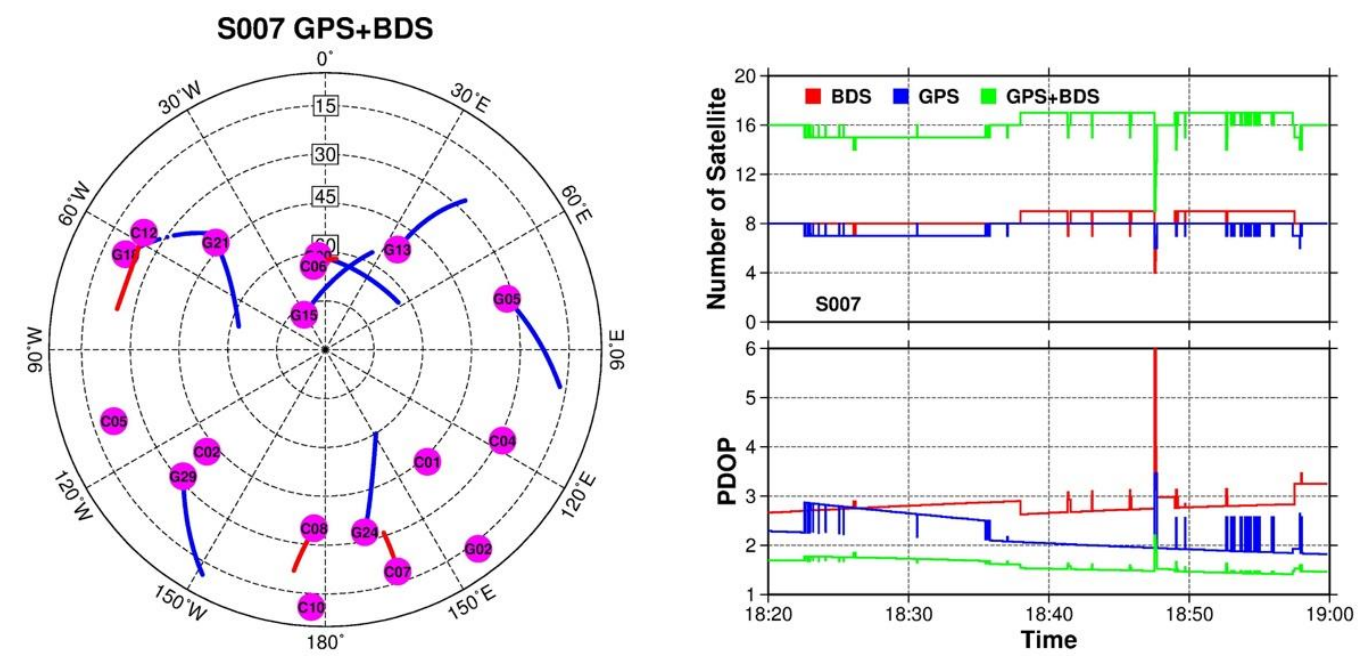

Fig 10 Sky plot of site S007 (Left panel). GPS satellites were denoted by the blue points and BDS satellites were the red points. The time scale of the experiment is from 18:20 to 19:00 at the local time. The satellite visibility of GPS and BDS satellite and corresponding PDOP value series for S007 (Right Panel).

S007 located at a box girder beside the western tower. From 18:20 to 19:00, a case of an obstruction phenomenon caused by tower and of an obstruction caused by passing vehicles. As seen in the left panel of Fig 10, the sky plot of S007, G18 had a same orientation with the western tower respect to the location of S007. The masking of satellite G18 by the tower occurred between 12:23 and 12:36. At 12:47, from the right panel of Fig 10, the number of GPS and BDS satellites were dropping instantaneously at the moment. Satellite number drop causes the satellite geometry (PDOP) change dramatically for GPS and BDS standalone system, and the observed satellites can even be lower to 4 for BDS at 12:47. However, the combination of GPS and BDS can have about 16 satellites observed during the whole session, and the PDOP value kept stable when one or two satellites were 
excluded. At 12:47, there were still 9 satellites can be tracked and the PDOP value did not change much compared with the standalone GPS and BDS. In this case, the monitoring availability can be improved with the integration of GPS and BDS.
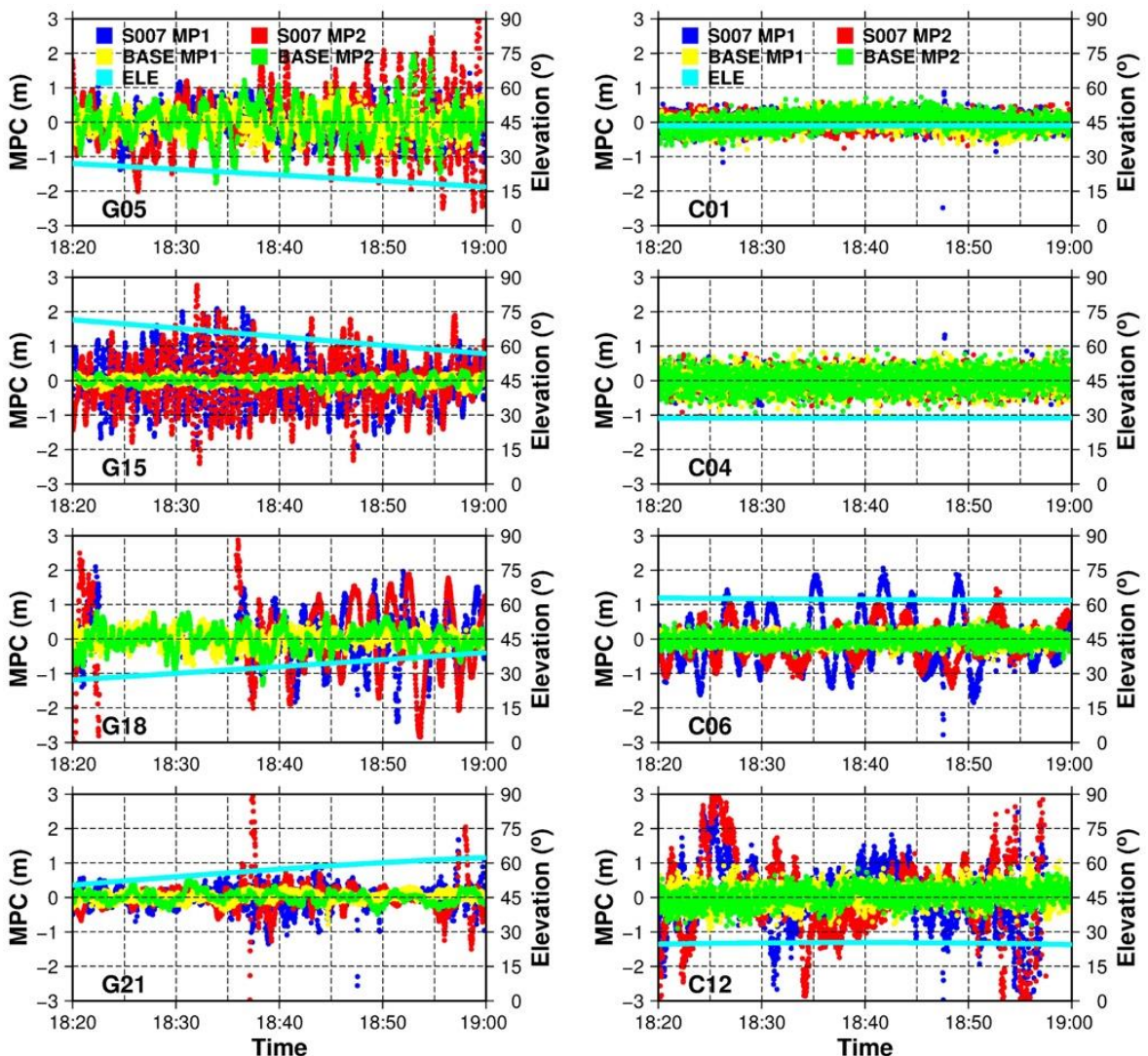

Fig 11 Multipath combinations of selected GPS (left panel) and BDS (right panel) satellites at S007 and BASE.

As previously discussed, the multipath effect and signal diffraction could also be severe in bridge monitoring environment. Fig 11 presents the time series of Multipath Combinations (MPC) (Paziewski and Sieradzki 2017; Shi et al. 2012) of S007 and the reference station BASE for selected GPS (left panel) and BDS satellites (right panel). It is evident, except for the two GEO satellites of BDS, the MPC of S007 is larger than BASE for other satellites, even when the elevation is higher than $60^{\circ}$ for G15 and C06. Meanwhile, Fig 12 shows the C/N0 time series of G18 and G21 at S007 and the reference station BASE. It can be seen that the C/NO time series in S007 changed dramatically, even for the high elevation satellites. However, the reference station always shows a stable time series. 

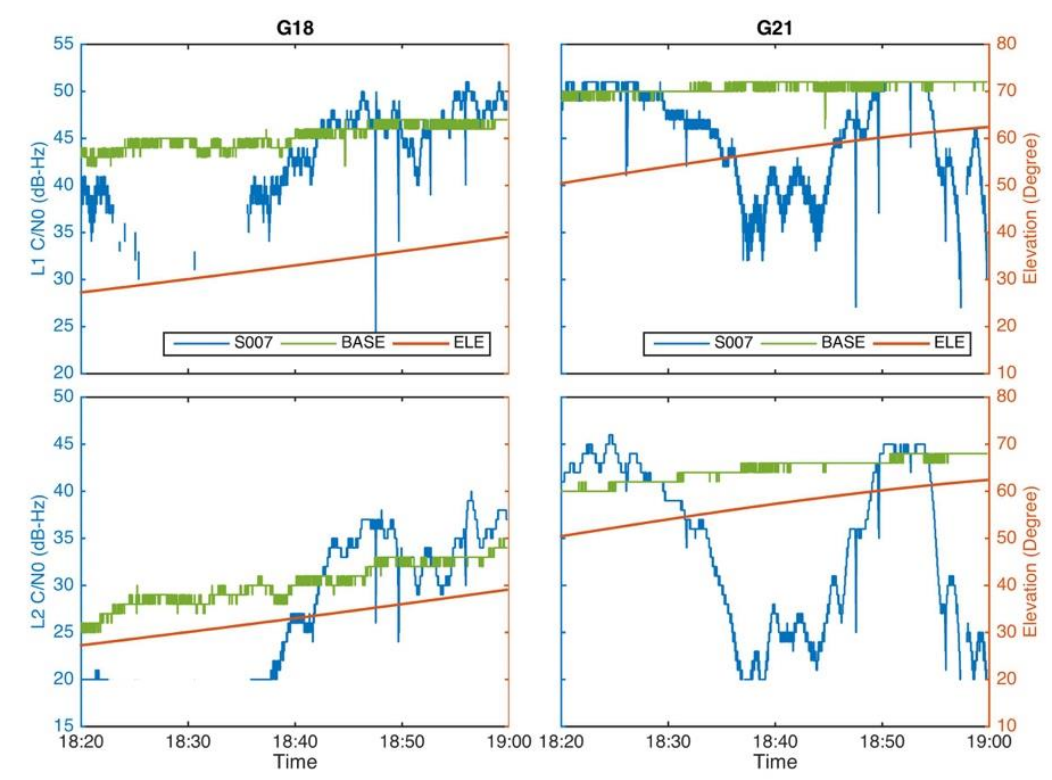

Fig $12 \mathrm{C} / \mathrm{N} 0$ and elevation time series for S007 and BASE station. The red line is satellite elevation time series during this moment.

In data processing, the observations of GPS and BDS can be biased by the multipath effect and signal diffraction caused by obstructions. It can not only affect the ambiguity resolution, but also introduce gross error. On the one hand, the ambiguity can be fixed to an incorrect integer number if the multipath effect errors were involved. On the other hand, if we still use the elevation stochastic model to weight the observations, the biased observations will be involved in the adjustment calculation under an inappropriate weight. Thus, the ambiguity checking procedure and SNR based stochastic model are suggested in this paper. To provide an effective comparison with monitoring performance, based on the $\mathrm{S} 007$ station, we processed the data in three schemes.

Scheme 1 GPS+BDS data processing with elevation stochastic model shown in Eq. (13) (Li and Zhang 2017), without post-fit residual editing for ambiguities checking;

$$
\sigma^{2}=m^{2}+m^{2} / \sin ^{2}(\theta)
$$

where $m$ is set to $3 \mathrm{~mm}$ and $\theta$ is elevation angle.

Scheme 2 GPS+BDS data processing with elevation stochastic model in Eq. (10), and with post-fit residual editing for ambiguities checking;

Scheme 3 GPS+BDS data processing with SNR stochastic model in Eq. (10), and with post-fit residual editing for ambiguities checking;

The results are listed in Fig 13. It can be clearly noticed that there are many incorrect biases in 
the result of Scheme 1. The sudden jumps are mainly happened when the ambiguities are fixed to the incorrect integer numbers. After ambiguities checking was applied, Scheme 2 shows a more reasonable solution. However, there are still many existing gross errors. For Scheme 3, the time series become more reasonable and reliable with SNR stochastic model in Eq. (10). The number of jumps are obviously reduced. Except for the big variations ahead of 18:47 and 18:57 (the satellite exclusion), the time series become smoother compared with Schemes 1 and 2.

Therefore, we know that the contribution of BDS can increase the satellite visibility and enhance the satellite geometry. The availability and reliability of bridge monitoring at obstruction environment can be effectively improved with SNR stochastic model and the post-fit residual editing procedure.

Scheme 1
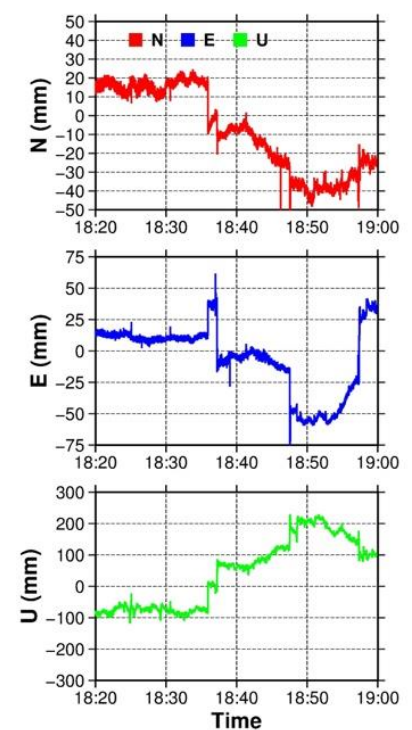

Scheme 2
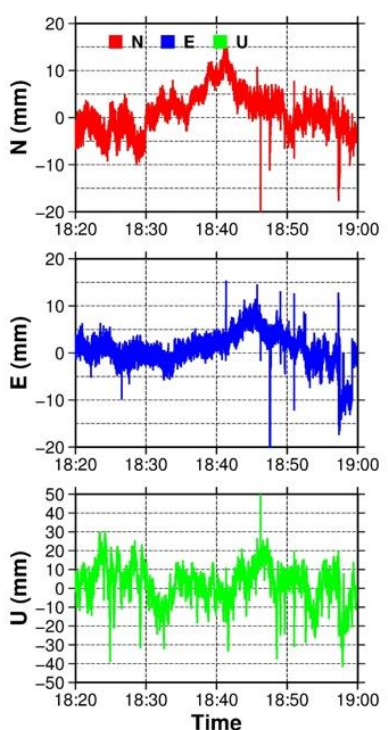

Scheme 3
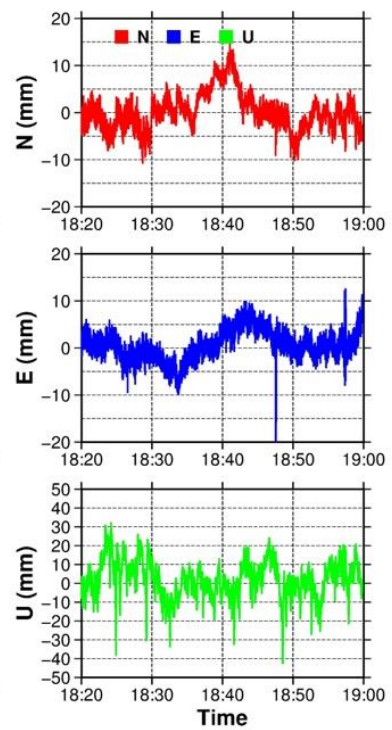

Fig 13 Time series of three schemes of data processing.

\section{Conclusions}

In this study, in order to improve the reliability of GNSS-based bridge monitoring technology, an integrated GPS and BDS data processing method with specific strategies, such as SNR-based stochastic model and post-fit residual editing procedure, was proposed. At a group of fixed points, the ambiguity resolution and precision performance of the proposed method were assessed. The ambiguity success fixing rate of GPS-only, BDS-only and GPS+BDS data can achieve to approximately $95 \%$ within only one epoch when there are no shelters around the stations. Compared with GPS-only results, an improvement of $20 \%$ to $30 \%$ in precision can be achieved with GPS+BDS 
data. Based on the real bridge monitoring data, the proposed method was assessed. The results indicate that, the introduction of BDS into the solution has little effect on natural frequencies identification at ideal observation environment. However, the combined GPS and BDS results seem to be much more promising, with lower background noise. Meanwhile, integrated GPS and BDS data processing with the SNR-based stochastic model and post-fit residual editing strategies can effectively deal with the satellite signal obstruction and the influence of multipath effect on ambiguity resolution, to attain reliable dynamic deformation monitoring information for bridges.

\section{Acknowledgment}

This work is supported by "the Open Foundation of Key Laboratory of Precise Engineering and Industry Surveying of National Administration of Surveying, Mapping and Geoinformation" (Grant No. PF2017-8). This research was also supported by the National Natural Science Foundation of China (Grant Nos. 41525014, 41704030 and 41210006), the Program for Changjiang Scholars of the Ministry of Education of China. The authors would like to express their gratitude to the European Space Agency for continuously sponsoring the GeoSHM Projects. Thanks also go to the China Scholarship Council (CSC) for the funding of the first author's living expenses during his study at the University of Nottingham in the UK. We are grateful to the anonymous reviewers and editors for their very constructive suggestions and comments that helped us to significantly improve the paper quality.

\section{References}

[1] Amiri-Simkooei A R, Tiberius C. Assessing receiver noise using GPS short baseline time series[J]. GPS solutions, 2007, 11(1): 21-35.

[2] Brunner F K, Hartinger H, Troyer L. GPS signal diffraction modelling: the stochastic SIGMA- $\Delta$ model[J]. Journal of Geodesy, 1999, 73(5): 259-267.

[3] Chan W S, Xu Y L, Ding X L, et al. An integrated GPS-accelerometer data processing technique for structural deformation monitoring[J]. Journal of Geodesy, 2006, 80(12): 705-719.

[4] Chen G. GPS kinematic positioning for the airborne laser altimetry at Long Valley, California[D]. Massachusetts Institute of Technology, 1998.

[5] DAI Wujiao, DING Xiaoli, ZHU jianjun. Comparing GPS Stochastic Models Based on Observation Quality Indices[J]. Geomatics and Information Science of Wuhan University, 2008, 
33(7): 718-722.

[6] Deng C, Tang W, Liu J, et al. Reliable single-epoch ambiguity resolution for short baselines using combined GPS/BeiDou system[J]. GPS solutions, 2014, 18(3): 375-386.

[7] GAO Xingwei, GUO Jingjun, CHENG Pengfei, et al.Fusion positioning of Compas/GPS Based on Spatio Temporal System Unification [J].Acta Geodaetica et Cartographica Sinica, 2012, 41(5):743-748, 755.

[8] Han H, Wang J, Meng X, et al. Analysis of the dynamic response of a long span bridge using GPS/accelerometer/anemometer under typhoon loading[J]. Engineering Structures, 2016, 122: 238-250.

[9] Hartinger H, Brunner F K. Variances of GPS phase observations: the SIGMA- $\varepsilon$ model[J]. GPS solutions, 1999, 2(4): 35-43.

[10] Herring T A, King R W, Floyd M A, et al. GAMIT-GPS Analysis at MIT, Reference Manual 10.6[J]. Department of Earth, Atmospheric, and Planetary Sciences Massachusetts Institute of Technology, 2015.

[11] Huang Shengxiang, Wu Wentan, Li Peihong. GPS Dynamic Monitoring Experiment and Analysis of Long-Span Cable-Stayed Bridge[J]. Geomatics and Information Science of Wuhan University, 2005, 30(11): 999-1002.

[12] Jiang W, Xi R, Chen H, et al. Accuracy analysis of continuous deformation monitoring using BeiDou Navigation Satellite System at middle and high latitudes in China[J]. Advances in Space Research, 2017, 59(3): 843-857.

[13] Lagler K, Schindelegger M, Böhm J, et al. GPT2: Empirical slant delay model for radio space geodetic techniques[J]. Geophysical research letters, 2013, 40(6): 1069-1073.

[14] Li P, Zhang X, Guo F. Ambiguity resolved precise point positioning with GPS and BeiDou[J]. Journal of Geodesy, 2017, 91(1): 25-40.

[15] Li P, Zhang X. Integrating GPS and GLONASS to accelerate convergence and initialization times of precise point positioning[J]. GPS solutions, 2014, 18(3): 461-471.

[16] Liu Hongfei. Research on Detection of Small Cycle Slips and Validation of Integer Ambiguities in GNSS Relative Positioning[D]. PhD dissertation. Wuhan University, 2013.

[17] Martín A, Anquela A B, Dimas-Pagés A, et al. Validation of performance of real-time kinematic PPP. A possible tool for deformation monitoring[J]. Measurement, 2015, 69: 95-108. 
[18] Meng X, Dodson A H, Roberts G W. Detecting bridge dynamics with GPS and triaxial accelerometers[J]. Engineering Structures, 2007, 29(11): 3178-3184.

[19] Meng X, Roberts G W, Cosser E, et al. Real-Time Bridge Deflection and Vibration Monitoring Using an Integrated GPS/Accelerometer/Pseudolite System[J]. 2003.

[20] Meng X, Roberts G W, Dodson A H, et al. Impact of GPS satellite and pseudolite geometry on structural deformation monitoring: analytical and empirical studies[J]. Journal of Geodesy, 2004, 77(12): 809-822.

[21] Moschas F, Psimoulis P A, Stiros S C. GPS/RTS data fusion to overcome signal deficiencies in certain bridge dynamic monitoring projects[J]. Smart Structures and Systems, 2013, 12(3_4): 251269.

[22] Moschas F, Stiros S. Dynamic multipath in structural bridge monitoring: an experimental approach[J]. GPS solutions, 2014, 18(2): 209-218.

[23] Moschas F, Stiros S. Measurement of the dynamic displacements and of the modal frequencies of a short-span pedestrian bridge using GPS and an accelerometer[J]. Engineering Structures, 2011, 33(1): 10-17.

[24] Msaewe H A, Hancock C M, Psimoulis P A, et al. Investigating multi-GNSS performance in the UK and China based on a zero-baseline measurement approach[J]. Measurement, 2017, 102: 186199.

[25] Paziewski J, Sieradzki R. Integrated GPS+ BDS instantaneous medium baseline RTK positioning: Signal analysis, methodology and performance assessment[J]. Advances in Space Research, 2017, 60(12): 2561-2573.

[26] Peppa I, Psimoulis P, Meng X. Using the signal - to - noise ratio of GPS records to detect motion of structures[J]. Structural Control and Health Monitoring, 2018, 25(2).

[27] Psimoulis P, Pytharouli S, Karambalis D, et al. Potential of Global Positioning System (GPS) to measure frequencies of oscillations of engineering structures[J]. Journal of Sound and Vibration, 2008, 318(3): 606-623.

[28] Roberts G W, Tang X. The use of PSD analysis on BeiDou and GPS $10 \mathrm{~Hz}$ dynamic data for change detection[J]. Advances in Space Research, 2016.

[29] Shi C, Zhao Q L, Li M, et al. Precise orbit determination of Beidou satellites with precise positioning[J]. Science China Earth Sciences, 2012, 55(7): 1079-1086. 
[30] Teunissen P J G. The least-squares ambiguity decorrelation adjustment: a method for fast GPS integer ambiguity estimation[J]. Journal of geodesy, 1995, 70(1): 65-82.

[31] Wang D, Meng X, Gao C, et al. Multipath extraction and mitigation for bridge deformation monitoring using a single-difference model[J]. Advances in Space Research, 2017.

[32] Wang J, Meng X, Qin C, et al. Vibration frequencies extraction of the forth road bridge using high sampling GPS data[J]. Shock and Vibration, 2015, 2016.

[33] Weber G, Mervart L. BKG Ntrip Client (BNC) Version 2.7 Manual[J]. Federal Agency for Cartography and Geodesy, Frankfurt, Germany, 2012.

[34] Wieser A, Brunner F K. Analysis of bridge deformations using continuous GPS measurements[C]//INGEO2002, 2nd Conference of Engineering Surveying. 2002: 8.

[35] Xi R, Jiang W, Chen Q, Meng X. Analysis of Bridge Deformations using Real-time BDS Measurements [C]//CPGPS 2017 Forum, 2017. (In press)

[36] Xi R, Xiao Y, Liu X, et al. Feasibility Analysis of High-Precision Deformation Monitoring Using BeiDou Navigation Satellite System[C]. China Satellite Navigation Conference (CSNC) 2015 Proceedings: Volume I. Springer Berlin Heidelberg, 2015: 27-34.

[37] Xiao Yugang, JIANG Weiping, CHEN Hua, et al. Research and Realization of Deformation Monitoring Algorithm with Milimeter Level Precision Based on BeiDou Navigation Satelite System[J]. Acta Geodaetica et Cartographica Sinica , $2016 \quad, \quad 45 \quad$ (1):1621.DOI:10.11947/j.AGCS.2016.20140649.

[38] Yang Y X, Li J L, Xu J Y, et al. Contribution of the compass satellite navigation system to global PNT users[J]. Chinese Science Bulletin, 2011, 56(26): 2813-2819.

[39] Yi T H, Li H N, Gu M. Experimental assessment of high-rate GPS receivers for deformation monitoring of bridge[J]. Measurement, 2013, 46(1): 420-432.

[40] Yu J, Meng X, Shao X, et al. Identification of dynamic displacements and modal frequencies of a medium-span suspension bridge using multimode GNSS processing[J]. Engineering Structures, 2014, 81: 432-443.

[41] Zhang Jinwu, Li Longan. Aseismic and wind-resistant analysis for Wuhan Baishazhou Yangtze River Bridge[J]. Bridge Construction, 1998, 3: 8-12. 